\title{
Trees with an On-Line Degree Ramsey Number of Four
}

\author{
David Rolnick \\ Massachusetts Institute of Technology \\ Cambridge, MA, USA \\ drolnick@mit.edu
}

Submitted: Dec 17, 2010; Accepted: Aug 11, 2011; Published: Sep 2, 2011

Mathematics Subject Classification: 05C55, 05C57, 05C05

\begin{abstract}
On-line Ramsey theory studies a graph-building game between two players. The player called Builder builds edges one at a time, and the player called Painter paints each new edge red or blue after it is built. The graph constructed is called the background graph. Builder's goal is to cause the background graph to contain a monochromatic copy of a given goal graph, and Painter's goal is to prevent this. In the $S_{k}$-game variant of the typical game, the background graph is constrained to have maximum degree no greater than $k$. The on-line degree Ramsey number $\stackrel{\circ}{R}_{\Delta}(G)$ of a graph $G$ is the minimum $k$ such that Builder wins an $S_{k}$-game in which $G$ is the goal graph. Butterfield et al. previously determined all graphs $G$ satisfying $\stackrel{\circ}{R}_{\Delta}(G) \leq 3$. We provide a complete classification of trees $T$ satisfying $\stackrel{\circ}{R}_{\Delta}(T)=4$.
\end{abstract}

\section{Introduction}

The quintessential problem of Ramsey theory involves finding a monochromatic copy of a graph $G$ within a larger graph whose edges are colored with some $s$ colors. Given $G$ and $s$, we say that a graph $H$ arrows $G$ if every $s$-coloring of $H$ contains a monochromatic $G$ as a subgraph. Two basic parameters of Ramsey theory are

- The Ramsey number $R(G)$ is the minimum number of vertices among graphs $H$ that arrow $G$.

- The size Ramsey number $\hat{R}(G)$ is the minimum number of edges among graphs that arrow $G$.

The numbers called on-line Ramsey numbers are based upon the following game, which we consider in the 2-color case, though an $s$-color generalization is possible: Two 
players, called Builder and Painter, generate a 2-colored graph $H$. Builder builds edges one at a time, using some combination of existing vertices and new vertices. As each edge is built, Painter paints it either red or blue. Builder's goal is for the graph $H$ to contain a monochromatic copy of some given graph $G$, and Painter's goal is to prevent this from happening. We will call the graph $G$ the goal graph and the 2-colored graph $H$ that is being built the background graph. Note that the background graph gets bigger throughout the game.

If Builder is allowed to build edges without constraint, eventually he can generate a "large enough" background graph that it contains a monochromatic copy of $G$ no matter how Painter has colored the edges. (This intuitively apparent result follows immediately from Ramsey's Theorem.) One may, however, consider the minimum number of edges Builder must draw before he wins. This number is the on-line size Ramsey number and is denoted $\tilde{r}(G)$. An important conjecture in this area is that

$$
\lim _{n \rightarrow \infty} \frac{\tilde{r}\left(K_{n}\right)}{\hat{R}\left(K_{n}\right)}=0
$$

where $\hat{R}(G)$ is the (standard) size Ramsey number. Recently, Conlon [2] has made significant progress towards proving this conjecture, by showing that the given limit is indeed 0 if $n$ is restricted to a certain subsequence of integers.

As an alternative to considering the size Ramsey number, the game can be modified so that Builder is allowed to build only edges such that the background graph remains within a specified class of graphs $\mathcal{H}$. We call such a game an $\mathcal{H}$-game.

We will consider the case where $\mathcal{H}$ is the class $S_{k}$ of graphs $H$ such that $\Delta(H) \leq k$, where $\Delta(H)$ denotes the maximum degree of the graph $H$. The on-line degree Ramsey number $R_{\Delta}(G)$ is defined to be the minimum $k$ such that Builder can win an $S_{k}$-game where $G$ is the goal graph.

Butterfield et al. [1] have studied $\stackrel{\circ}{R}_{\Delta}(G)$. Among their results are the following:

- $\stackrel{\circ}{R}_{\Delta}\left(K_{1, n}\right)=n$ for stars $K_{1, n}$.

- The graphs $G$ for which $\stackrel{\circ}{R}_{\Delta}(G) \leq 3$ are exactly those graphs for which either (i) every component is a path, or (ii) every component is a subgraph of the claw $K_{1,3}$.

- For all $G$,

$$
\stackrel{\circ}{R}_{\Delta}(G) \geq \Delta(G)-1+\max _{u v \in E(G)} \min \{d(u), d(v)\}
$$

- If $G$ is a tree with $d_{1} \geq d_{2}$ being its greatest two degrees, then

$$
\stackrel{\circ}{R}_{\Delta}(G) \leq d_{1}+d_{2}-1
$$

with equality if $G$ has adjacent vertices of degrees $d_{1}$ and $d_{2}$.

Few graphs with on-line degree Ramsey number greater than 3 have hitherto been identified. In this paper, we present the following classification of trees $T$ with $\stackrel{\circ}{R}_{\Delta}(T)=4$ :

Let $M$ denote the set of trees with maximum degree at most 3 and with no two adjacent degree-3 vertices. Then, the set of trees $T$ for which $\stackrel{\circ}{R}_{\Delta}(T)=4$ is the set $M$, with the addition of the graph $K_{1,4}$ and with the removal of the claw $K_{1,3}$ and of all paths $P_{n}$. 


\section{Definitions}

Given a subgraph $H_{0}$ of a given graph $H$, we will say that $H_{0}$ is isolated if it is its own component.

A Painter is called consistent if, whenever he is given a new edge $e$ to be added to a background graph $H$, the color he assigns to $e$ depends only on that component of $H \cup\{e\}$ containing $e$. Thus, for instance, a consistent Painter, when presented by Builder with an isolated edge, will always color it the same way, regardless of the other components of the background graph.

It is proven in [1] that, for any graph $G$ and integer $k$, Builder can win a $S_{k}$-game if and only if Builder can win against a consistent Painter. Hence, for the remainder of this paper, we will assume that all Painters are consistent.

Following [1], we define a weight function on a graph $G$ to be a function $f$ assigning a positive integer to each vertex. A weighted graph is a graph $G$ with an associated weight function. Given a weighted graph $G$ we say that a (non-weighted) graph $H$ contains $G$ if $H$ contains as a subgraph a non-weighted copy $G^{\prime}$ of $G$ with the weight of each vertex of $G$ being no less than the degree in $H$ of the corresponding vertex of $G^{\prime}$. In general, when we speak of "graphs" in this paper, we will mean weighted graphs.

Let a maple be a tree with maximum degree at most 3 and with no two adjacent degree-3 vertices. Let a fork within a maple be a degree-3 vertex. We say that a vertex $v$ abuts a given fork $y$ in the same maple if the path joining $v$ and $y$ does not include any forks (except $y$ and possibly $v$ ).

\section{Results}

Our goal in this section will be to prove the folowing theorem:

Theorem 3.1. The set of trees $T$ having $\stackrel{\circ}{R}_{\Delta}(T) \leq 4$ is the set of maples, with the addition of the graph $K_{1,4}$.

It is proven in [1] that, for any graph $G$,

$$
\stackrel{\circ}{R}_{\Delta}(G) \geq \Delta(G)-1+\max _{u v \in E(G)} \min \{d(u), d(v)\}
$$

An immediate corollary of this result is that if $T$ is a tree with $\stackrel{\circ}{R}_{\Delta}(T) \leq 4$, then $T$ must be either a maple or the graph $K_{1,4}$. Since $\stackrel{\circ}{R}_{\Delta}\left(K_{1, n}\right)=n$ is proven in [1], to prove Theorem 3.1 it suffices to prove that Builder can win an $S_{4}$-game with goal graph some fixed maple. Consider then an $S_{4}$-game with goal graph some maple $T$. Suppose towards contradiction that Painter never forms a monochromatic $T$. Without loss of generality, assume that our consistent Painter colors any isolated edge red. Our strategy in this proof is to show that Builder can construct a red copy of $T$.

Suppose that Builder can force some (2-colored) graph $H$, with $v$ a vertex of $H$ having weight 1 . Then, we claim that the following four results hold: 
Tree Extension Lemma. Builder can force the (weighted, 2-colored) graph shown in Figure 1(a).

2-2 Branching Lemma. Builder can force the graph shown in Figure 1(b).

3-3 Branching Lemma. Builder can force the graph shown in Figure 1(c).

2-3 Branching Lemma. Builder can force the graph shown in Figure 1(d).

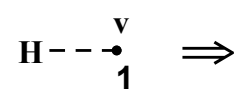

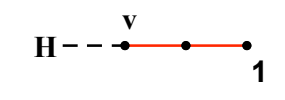

(a)

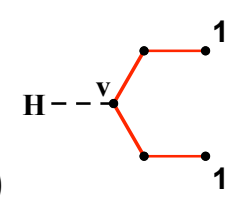

(c)

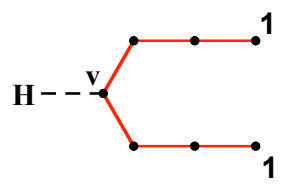

(d)

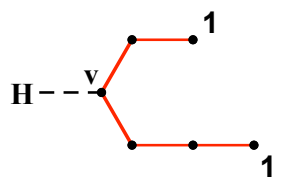

Figure 1: (a) The Tree Extension Lemma, (b) the 2-2 Branching Lemma, (c) the 33 Branching Lemma, (d) the 2-3 Branching Lemma. The numerals beside some of the vertices indicate weights, with unnumbered vertices assumed to have the maximum possible weight of 4 .

The majority of our proof will be devoted to proving that these lemmas hold. We begin with the Tree Extension Lemma, which will be proven as a corollary of the following result.

Claim 3.2. Suppose Builder can force a copy of the (2-colored) graphs $J$ and $K$, with $w$ a vertex of $J$ having weight 1 and $u$ a vertex of $K$ having weight 1 . Then, she can force one of the two graphs shown in Figure 2(a).

(a)
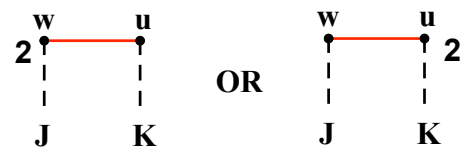

Figure 2: (a) The desired (red) connections between $J$ and $K$, (b) forcing a blue $T$ if the desired red connections are avoided. (Blue edges are shown thicker than red for convenience in gray-scale viewing.)

Proof. Taking advantage of the consistency of our Painter, Builder constructs many copies each of $J$ and $K$. She then attempts to construct a blue copy of $T$ as follows (see Figure $2(\mathrm{~b}))$. 
For each vertex of $T$, Builder picks a copy of either $J$ or $K$ such that if two vertices of $T$ are adjacent, one vertex is represented by $J$ and the other by $K$. For each copy of $J$ or $K$ thus chosen, the vertex $w$ or $u$ respectively is called the apex. Then, she puts all the edges of $T$ in a sequence: She starts with an edge incident to a leaf of $T$; she then adds edges successively so that any prefix subsequence of edges forms a connected subgraph of $T$. Builder then moves through the sequence, reading off edges. As she reads off each edge $e$, she connects the respective apexes of the copies of $J$ and $K$ representing the endpoints of $e$. If any such connecting edge is red, then one of the graphs depicted in Figure 2(a) must be formed. Otherwise, all connecting edges are blue, and Builder eventually forces a blue copy of $T$.

Corollary 3.3. Suppose Builder can force copies of the (2-colored) graphs $J$ and $K$, with $w$ a vertex of $J$ having weight 1 and $u$ a vertex of $K$ having weight 1 . Then, she can force the graph shown in Figure 3(a), with $J$ and $K$ joined by a red edge incident to $w$ and $u$.

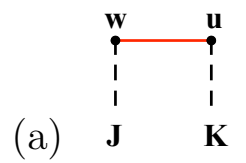

Figure 3: (a) The graph desired in Corollary 3.3, (b) the graphs desired in Claim 3.4.

The Tree Extension Lemma follows from the above corollary if we let $J$ be $H, w$ be $v$, and $K$ be an isolated red edge.

Before proving the 2-2 Branching Lemma, we must prove several more results.

Claim 3.4. Suppose Builder can force copies of the (2-colored) graphs $J$ and $K$, with $w$ a vertex of $J$ having weight 2 and $u$ a vertex of $K$ having weight 1 . Then, she can force one of the two graphs shown in Figure 3(b).

Proof. For each vertex of $T$, Builder picks a copy of either $J$ or $K$, with $J$ chosen if the vertex has degree 1 or 2 and $K$ chosen if the vertex is a fork (i.e., has degree 3). For each copy of $J$ or $K$ thus chosen, the vertex $w$ or $u$ respectively is called the apex. Then, for every two adjacent vertices of $T$, Builder connects the respective apexes of the two graphs representing the vertices. If any connecting edge is red, the claim is proven, since the edge must run either between the apex of a $J$ and the apex of a $K$ or else between the apexes of two copies of $J$. Otherwise, all the connecting edges are blue, and Builder has made a blue copy of $T$.

We call a subtree $U$ of $T$ fitting if $T$ and $U$ share a leaf and every fork in $T$ that is a vertex of $U$ is either a leaf or a fork of $U$.

Claim 3.5. Builder can force the weighted red-blue-red path shown in Figure 4(a). 
(a)

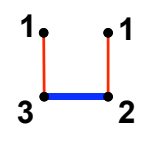

(b)

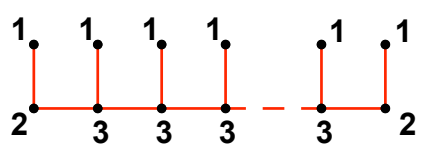

Figure 4: (a) The graph desired in Claim 3.5, (b) a comb.

Proof. Builder begins by constructing numerous copies of an isolated red edge $a b$ and stringing them together by repeatedly connecting the $b$ of the last $a b$ in the chain to the $b$ of a new copy of $a b$. If any of the connecting edges is blue, then the claim is proven. Otherwise, Builder can force an arbitrarily long red chain of the form shown in Figure 4(b). We call such a chain a comb, and let the spine of a comb refer to the path joining the two degree 2 vertices. Vertices not in the spine shall be referred to as teeth. It suffices to show that arbitrarily long combs allow Builder to win.

Suppose Builder can force arbitrarily long combs. We show that she can use the combs to construct a red copy of $T$. Such a copy of $T$ may be expressed by an injective function $f$ from $V(T)$ to the vertex set of the background graph of the game, such that two adjacent vertices in $V(T)$ are sent to vertices adjacent by a red edge.

Builder defines such a function progressively over $V(T)$. Builder starts at some leaf $\ell$ of the maple $T$, making its image under $f$ be some spine vertex of a sufficiently large comb. If $\ell$ is at distance $d$ from the nearest fork $a$ in $T$, Builder then moves along the spine of the comb in some direction, making each new spine vertex the image of a vertex in $T$ as adjacency warrants, until she has moved $d$ edges along the spine and makes the spine vertex she has reached be the image of the fork $a$ of $T$. She then proceeds to define elements of $f(V(T))$ in succession. At any given point in this process, and for any fork $y$ whose image $f(y)$ has been defined, we say that a $y$-successor fork is a fork of $T$ that abuts $y$ and whose image has not yet been defined.

Assume that Builder has already defined $f(V(U))$ for some fitting subtree $U$ of $T$, where, for every fork $y$ of $T$ that is a leaf of $U$, the following conditions hold:

- There is some subgraph $L(y)$ of the background graph isomorphic to a (sufficiently large) unweighted comb and containing $f(y)$ as a spine vertex.

- The tooth of $L(y)$ adjacent to $f(y)$ has degree 1 and is not in the image $f(V(U))$.

- Of the two pieces into which $f(y)$ divides the spine of $L(y)$, one piece, together with all teeth coming off of it, is sufficiently large and is unused - that is, the degrees of its vertices in the background graph are no higher than they would be in an isolated (weighted) comb, and none of the vertices are in the image $f(V(U))$.

Now consider some fork $y_{0}$ of $T$ that is a leaf of $U$. Let $L_{0}=L\left(y_{0}\right)$.

If there are no $y_{0}$-successor forks, then Builder moves along the spine of $L_{0}$ in the unused direction. As she passes over each spine vertex, she makes it the image of a vertex of $T$ as adjacency warrants, and finally reaches the image of a leaf of $T$. To obtain 
the image of the other leaf abutting $y_{0}$, Builder uses repeated applications of the Tree Extension Lemma to append a long red path to the tooth adjacent to $y_{0}$. She then moves along the tooth and appended path, assigning images as adjacency warrants until she reaches the image of the leaf.

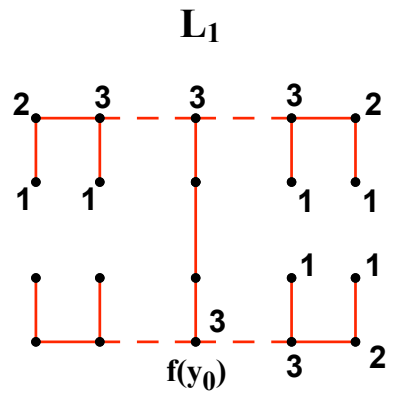

$\mathbf{L}_{0}$

Figure 5: Connecting the combs $L_{0}$ and $L_{1}$.

If there is exactly one $y_{0}$-successor fork $y_{1}$ at distance $d_{1}$ from $y_{0}$, then Builder moves $d_{1}$ edges, one by one, along the spine of $L_{0}$ in the unused direction. As she passes over each spine vertex, she makes it the image of a vertex of $T$ as adjacency warrants. After moving $d_{1}$ edges from $f\left(y_{0}\right)$, she lets $f\left(y_{1}\right)$ be the vertex she has reached. To obtain the image of the leaf abutting $y_{0}$, Builder again uses repeated applications of the Tree Extension Lemma to append a long red path to the tooth adjacent to $y_{0}$ and then assigns images along this path as adjacency warrants until reaching the image of the leaf. She now continues recursively as above, using $y_{1}$ in place of $y_{0}$.

Now suppose that there are two $y_{0}$-successor forks $y_{1}$ and $y_{2}$, at distances $d_{1}$ and $d_{2}$, respectively, from $y_{0}$. There are two cases to be considered.

Case 3.5.1. $d_{2} \geq 3$.

In this case, Builder forces a new comb $L_{1}$ and, using Corollary 3.3, connects one of its teeth via a red edge to the tooth of $L_{0}$ adjacent to $f\left(y_{0}\right)$ (see Figure 5). Builder moves along teeth from $L_{0}$ to $L_{1}$ and then along the spine of $L_{1}$, assigning images to vertices of $T$ as adjacency warrants. When she has moved $d_{2}$ edges away from $f\left(y_{0}\right)$, she lets $f\left(y_{2}\right)$ be the vertex she has reached. If $d_{2}>3$, then she will have assigned $f(V(U))$ for a subtree $U$ of $T$ as our conditions required, and she continues recursively as above.

If $d_{2}=3$, then $f\left(y_{2}\right)$ is the only vertex on the spine of $L_{1}$ that has yet been assigned to $f\left(V(T)\right.$ ). Builder now moves along the spine of $L_{1}$ in each possible direction in turn, assigning vertices to $f(V(T))$ until she reaches the image of a fork (or a leaf), at which point she continues recursively as above (or stops).

Case 3.5.2. $d_{2}=2$. 
Let the background graph obtained up until this point be $Q$. Now, Builder forces a new comb $L_{1}$ and, using Claim 3.2, connects one of its teeth by a red edge to the tooth of $L_{0}$ adjacent to $f\left(y_{0}\right)$, generating either graph $A$ or $B$ as shown in Figure 6(a), where the vertices $x_{0}$ in $A$ and $B$ are as shown.

Sub-case 3.5.2.1. Graph $A$ is obtained.

Builder forces another new comb $L_{2}$, and, using Claim 3.4, either doubles $A$ at $x_{0}$ or else connects $A$ by $x_{0}$ to one of the teeth of $L_{2}$. One of the graphs $A_{1}$ or $A_{2}$ shown in Figure 6(b) must be obtained. Let $x_{1}, x_{2}, x_{3}, x_{0}^{\prime}, x_{1}^{\prime}, L_{0}^{\prime}$, and $L_{1}^{\prime}$ be as shown.

Suppose first that $A_{1}$ is obtained. Then, Builder lets $f\left(y_{2}\right)$ be $x_{0}$. If there are no $y_{2}$-successor forks, then Builder continues through $x_{1}$ into $L_{1}$ and along the spine, and through $x_{0}^{\prime}$ into $L_{1}^{\prime}$ and along the spine, assigning elements of $f(V(T))$ until assignment is complete.

If there is exactly one $y_{2}$-successor fork $y_{3}$, then Builder continues through $x_{1}$ into $L_{1}$ and along the spine, assigning elements of $f(V(T))$ until she reaches $f\left(y_{3}\right)$. She also moves from $x_{0}$ through $x_{0}^{\prime}$ into $L_{1}^{\prime}$, assigning elements of $f(V(T))$ until she reaches a leaf.

If there are two $y_{2}$-successor forks $y_{3}$ and $y_{4}$, then Builder continues through $x_{1}$ into $L_{1}$ and along the spine, assigning elements of $f(V(T))$ until she reaches $f\left(y_{3}\right)$ on the spine of $L_{1}$. She also moves from $x_{0}$ through $x_{0}^{\prime}$ into $L_{1}^{\prime}$ and along the spine, assigning elements of $f(V(T))$ until she reaches $f\left(y_{4}\right)$. If $f\left(y_{4}\right)=x_{1}^{\prime}$, she then moves along the spine of $L_{1}^{\prime}$ in each possible direction in turn, assigning vertices to $f(V(T))$ until she reaches the image of a fork (or a leaf), at which point she continues recursively as above (or stops).

If $A_{2}$ is obtained, Builder goes through exactly the same process as for $A_{1}$, with $L_{2}$, $x_{2}$, and $x_{3}$ replacing $L_{1}^{\prime}, x_{0}^{\prime}$, and $x_{1}^{\prime}$, respectively, throughout.

Sub-case 3.5.2.2. Graph $B$ is obtained.

Builder first forces a copy $B^{\prime}$ of $B$, having $x_{0}^{\prime}$ in place of $x_{0}$. She then forces a copy $Q^{\prime}$ of the previously obtained background graph $Q$, having vertex $x_{2}$ in place of the vertex $f\left(y_{0}\right)$ and with $x_{3}$ the leaf adjacent to $x_{2}$. Using Claim 3.4, Builder either connects $B$ at $x_{0}$ by a red edge with its copy $B^{\prime}$ at $x_{0}^{\prime}$ or else connects the copy $B^{\prime}$ at $x_{0}^{\prime}$ by a red edge to $Q^{\prime}$ at $x_{3}$. One of the graphs $B_{1}$ or $B_{2}$ shown in Figure $6(\mathrm{c})$ must be obtained. Let $x_{1}$, $x_{1}^{\prime}, L_{0}^{\prime}$, and $L_{1}^{\prime}$ be as shown.

Suppose first that $B_{1}$ is obtained. Then, Builder lets $f\left(y_{2}\right)$ be $x_{0}^{\prime}$. If there are no $y_{2}$-successor forks, then Builder continues into the unused half of $L_{0}^{\prime}$ and also through $x_{1}^{\prime}$ into $L_{1}$ and along the spine, assigning elements of $f(V(T))$ until assignment is complete.

If there is some $y_{2}$-successor fork $y_{3}$, then Builder continues through $x_{1}^{\prime}$ into $L_{1}^{\prime}$ and along the spine, assigning elements of $f(V(T))$ until she reaches $f\left(y_{3}\right)$. If $f\left(y_{3}\right)=x_{1}^{\prime}$, she then moves along the spine of $L_{1}^{\prime}$ in each possible direction in turn, assigning vertices to $f(V(T))$ until she reaches the image of a fork (or a leaf), at which point she continues recursively as before (or stops). Following assignment of $f\left(y_{3}\right)$, Builder moves from $x_{0}^{\prime}$ into the unused half of $L_{0}^{\prime}$, assigning elements of $f(V(T))$ until she reaches the image of another $y_{2}$-successor fork (or a leaf), at which point she continues recursively as above (or stops). 
Suppose now that $B_{2}$ is obtained. In this case, Builder reassigns from $B$ to $Q^{\prime}$ the elements of $f(V(T))$ which she has hitherto defined, so that, for instance, $f\left(y_{0}\right)$ now equals $x_{2}$. Proof continues as when $B_{1}$ is obtained.

The sub-case and case are finally complete.

To complete the recursive step, Builder assigns the images of $y_{1}$ and of the vertices on the path between $y_{0}$ and $y_{1}$ to vertices on the unused half of the spine of $L_{0}$.

A:

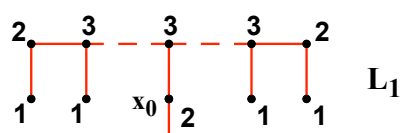

(a)

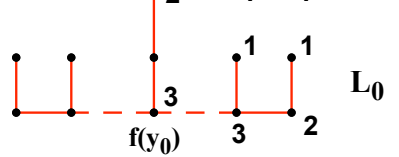

B:

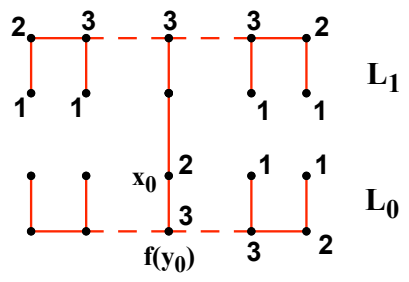

$\mathbf{A}_{1}$ :

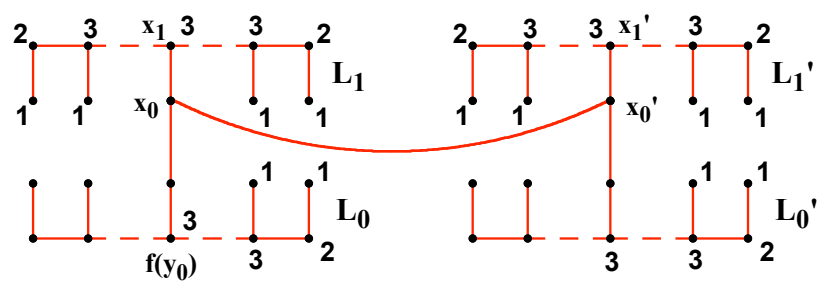

A2:

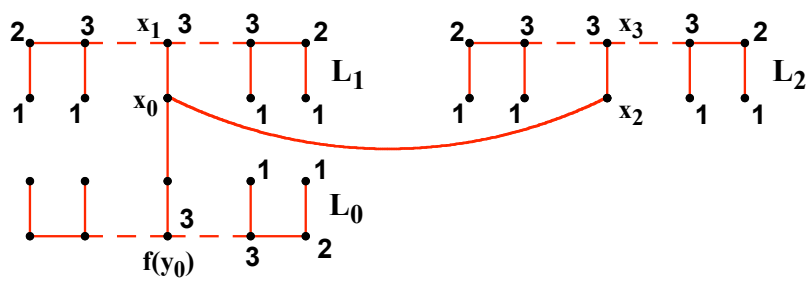

(b)

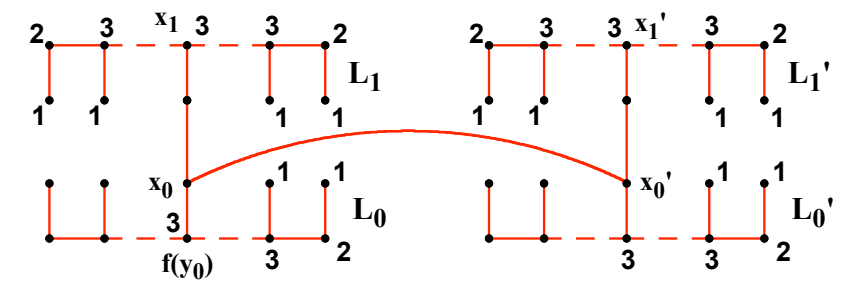

(c)

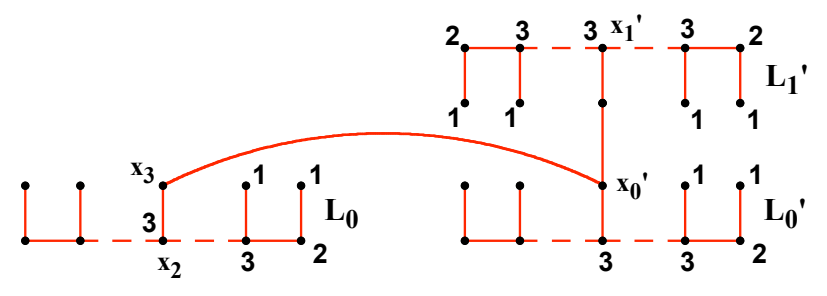

Figure 6: (a) The graphs $A$ and $B$, (b) the graphs $A_{1}$ and $A_{2}$, (c) the graphs $B_{1}$ and $B_{2}$.

We label as $x$ and $y$ two of the vertices of the graph $X$ in Figure 4(a), as shown in Figure 7(a).

Claim 3.6. Suppose that Builder can force some (2-colored) graph $H^{\prime}$, with $v^{\prime}$ a vertex of $H^{\prime}$ having weight 2. Then Builder can force the graph shown in Figure 7(b).

Proof. Builder forces many copies of the graph $H^{\prime}$, of the graph $X$, and of isolated (red) edges $a b$. The vertices $v^{\prime}$ in $H^{\prime}, x$ and $y$ in $X$, and $a$ in edges $a b$ are all called apexes. She 
(a)

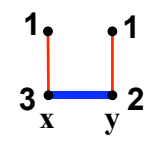

(b)

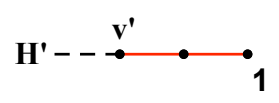

Figure 7: (a) Graph $X$ with vertices $x$ and $y$ marked, (b) the graph desired in Claim 3.6.

puts all the vertices of $T$ in a sequence $S=w_{1}, w_{2}, \ldots$ as follows: starting at a leaf $\ell$ of $T$, she successively adds vertices so that any prefix subsequence forms the vertex set of a connected subtree of $T$.

Builder now reads off this sequence, picking an apex $g\left(w_{n}\right)$ for each entry $w_{n}$ and connecting each apex to a previously chosen entry so that $g\left(w_{i}\right), g\left(w_{j}\right)$ are connected if and only if $w_{i}, w_{j}$ are. The kind of apex she chooses for the various entries of the sequence are as follows.

Builder picks an apex of the form $v^{\prime}$ for the image $g\left(w_{1}\right)$ of the first entry of $S$. Now consider $w_{n}$, for some $n \geq 2$. We know that $w_{n}$ must be adjacent to some (unique) vertex $w_{m}$ with $m<n$. Then, Builder picks $g\left(w_{n}\right)$ to be of the form

- $v^{\prime}$ if $g\left(w_{m}\right)$ is of the form $y$ or $a$.

- $x$ if $g\left(w_{m}\right)$ is of the form $v^{\prime}$ and the vertex $w_{n}$ is adjacent to a fork $w_{p}$ with $n<p$.

- $y$ if $g\left(w_{m}\right)$ is of the form $x$ - in this case, $g\left(w_{m}\right)$ and $g\left(w_{n}\right)$ should be apexes of the same copy of $X$ and will therefore already be connected by a (blue) edge.

- $a$ if $g\left(w_{m}\right)$ is of the form $v^{\prime}$ and if the vertex $w_{n}$ is not adjacent to any fork $w_{p}$ such that $n<p$.

It is readily verified that none of the edges between apexes can be red without yielding the graph in Figure 7(b). But if all these edges are blue, a blue $T$ is formed. Hence, the claim is proven.

Proof of the 2-2 Branching Lemma. Builder now forces many copies of the graph $H$, of the graph $X$, and of isolated (red) edges $a b$. The vertices $v$ in $H, x$ and $y$ in $X$, and $a$ in edges $a b$ are all called apexes. Again, Builder puts all the vertices of $T$ in a sequence $S=w_{1}, w_{2}, \ldots$ as follows: starting at one leaf $\ell$ of $T$, she successively adds vertices so that any prefix subsequence forms the vertex set of a connected subtree of $T$.

Builder now reads off this sequence, picking an apex $g\left(w_{n}\right)$ for each entry $w_{n}$ and connecting each apex to a previously chosen entry so that $g\left(w_{i}\right), g\left(w_{j}\right)$ are connected if and only if $w_{i}, w_{j}$ are. The kind of apex she chooses for the various entries of the sequence are as follows.

Builder picks an apex of the form $v$ for the image $g\left(w_{1}\right)$ of the first entry of $S$. Now consider $w_{n}$, for some $n \geq 2$. We know that $w_{n}$ must be adjacent to some vertex $w_{m}$ with $m<n$. Then, Builder picks $g\left(w_{n}\right)$ to be of the form

- $v$ if $g\left(w_{m}\right)$ is of the form $y$ or $a$. 
- $x$ if $g\left(w_{m}\right)$ is of the form $v$ and the vertex $w_{n}$ is adjacent to a fork $w_{p}$ with $n<p$.

- $y$ if $g\left(w_{m}\right)$ is of the form $x$ - in this case, $g\left(w_{m}\right)$ and $g\left(w_{n}\right)$ should be apexes of the same copy of $X$ and will therefore already be connected by a (blue) edge.

- $a$ if $g\left(w_{m}\right)$ is of the form $v$ and if the vertex $w_{n}$ is not adjacent to any fork $w_{p}$ such that $n<p$.

Builder stops connecting apexes if at any point the edge between two apexes is colored red by Painter, which must happen at some point if Painter is to prevent the formation of a blue $T$. Consider such a stoppage, precipitated by the red edge between $g\left(w_{\alpha}\right)$ and $g\left(w_{\beta}\right)$, where $\alpha<\beta$.

(*) If $g\left(w_{\beta}\right)$ is of the form $v$, then applying Claim 3.6 with $g\left(w_{\beta}\right)$ as $v^{\prime}$ proves the 2-2 Branching Lemma.

If $g\left(w_{\beta}\right)$ is not of the form $v$, then $g\left(w_{\alpha}\right)$ must be of the form $v$. In this case, Builder creates an apex of the same apex type as $g\left(w_{\beta}\right)$ and connects it to $g\left(w_{\alpha}\right)$, reassigning the label $g\left(w_{\beta}\right)$ to the new apex. If the edge between $g\left(w_{\alpha}\right)$ and the new $g\left(w_{\beta}\right)$ is red, then the 2-2 Branching Lemma is proven. Otherwise, any red-edge stoppage is moved to further along the sequence $S$.

Since the sequence $S$ is finite and stoppages cannot be pushed infinitely far along it, the 2-2 Branching Lemma must hold if a blue $T$ is to be prevented.

Before proving the final two branching lemmas, we prove some additional results.

Let graphs $L, M$, and $N$ be as shown in Figure 8(a).

(a)
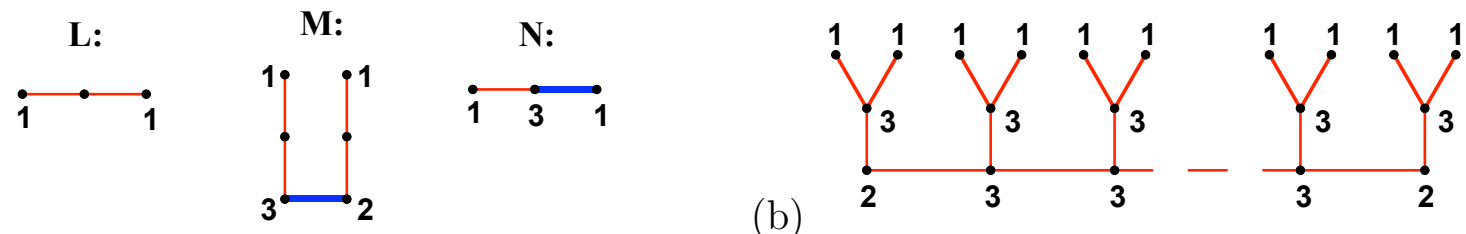

Figure 8: (a) The graphs $L, M$, and $N$, (b) a multiclaw.

Claim 3.7. Builder can force $L$.

Proof. Builder tries to construct a blue T. Assume that she has constructed some blue fitting subtree $U$ of $T$ such that all leaves of $U$ are of weight 1. Then, given a leaf $v$ of $U$, Builder draws three edges from it to new vertices. If at least two of the new edges are red, $L$ is obtained. Otherwise, at least two of the three edges are blue and so $U$ is enlarged. Hence, if a blue $T$ is to be prevented, Builder must be able to force $L$.

Claim 3.8. Builder can force either $M$ or $N$. 
Proof. We assume towards contradiction that Builder can force neither $M$ nor $N$.

Builder begins by constructing an isolated (red) edge, then connecting one endpoint of this edge to two new vertices. Since Builder cannot force $N$, Painter must color both these new edges red, showing that Builder can force a red claw with weight 3 in the center and weight 1 on each leaf. We let $C$ denote this (2-colored weighted) claw.

Define a multiclaw to be a graph of the form shown in Figure 8(b), obtained by stringing together many copies of $C$. Let the spine of a multiclaw be the long subpath depicted in Figure 8(b) as extending horizontally along the length of the multiclaw. We define the length of a multiclaw to be the number of edges in the spine.

Builder can force an arbitrarily long multiclaw as follows: She starts with $C$ (a length0 multiclaw). If she has already forced a length- $n$ multiclaw, she can force a length- $(n+1)$ multiclaw by connecting a terminal spine vertex of the multiclaw she has made to a leaf of a new copy of $C$. If the connecting edge is blue, then the graph $M$ is formed, so all such connecting edges must be red.

Now, we show that Builder can force a red copy of $T$. Such a copy of $T$ may be expressed by an injective function $h$ from $V(T)$ to the vertex set of the background graph of the game, such that two adjacent vertices in $V(T)$ are send to vertices adjacent by a red edge.

Builder defines such a function progressively over $V(T)$. She starts at some leaf of the maple $T$, making its image under $h$ be some spine vertex of a long multiclaw. If the chosen leaf is at distance $d$ from its nearest fork $a$ in $T$, Builder then moves along the spine of the multiclaw in some direction, making each new spine vertex the image of a vertex in $T$ as adjacency warrants, until she has moved $d$ edges along the spine and reached $h(a)$. She then proceeds to define elements of $h(V(T))$ in succession. At any given point in this process, and for any fork $y$ whose image $h(y)$ has been defined, we say that a $y$-successor is a fork or leaf of $T$ that abuts $y$ and whose image has not yet been defined.

Assume that Builder has already defined $h(V(U))$ for some fitting subtree $U$ of $T$, where for any fork $y$ of $T$ that is a leaf of $U$, the following conditions hold:

- As, shown in Figure 9(a), $h(y)$ is connected by red edges both to a multiclaw and to a red $P_{3}$ having weight 1 at its endpoints and weight 2 at its center.

- Both this multiclaw, which we call $Z(y)$, and this $P_{3}$, which we call $P(y)$, are disjoint from $h(V(U))$.

Now consider some fork $y_{0}$ of $T$ that is a leaf of $U$. Let $y_{1}$ and $y_{2}$ be the two successors of $y_{0}$, at distances $d_{1}$ and $d_{2}$ from $y_{0}$, respectively.

Builder first moves along the spine of $Z\left(y_{0}\right)$, assigning images to elements of $V(T)$ as adjacency warrants, until she has moved distance $d_{1}$ from $h\left(y_{0}\right)$ and reached $h\left(y_{1}\right)$. If $y_{1}$ is a leaf, then she stops, if a fork, then she can proceed as above, using $y_{1}$ in place of $y_{0}$.

To assign $h\left(y_{2}\right)$, Builder does as follows: She takes a leaf $r$ of $P\left(y_{0}\right)$ and attaches it to a leaf of a new copy of $C$, then to a leaf of another new copy of $C$. Both connecting edges must be red to prevent the formation of $M$. Builder then continues to add new copies of $C$, one by one, to the copies $C$ she has just attached, so that $r$ becomes connected to two multiclaws $Z_{1}$ and $Z_{2}$ of large length, as shown in Figure 9(b). 
(a)

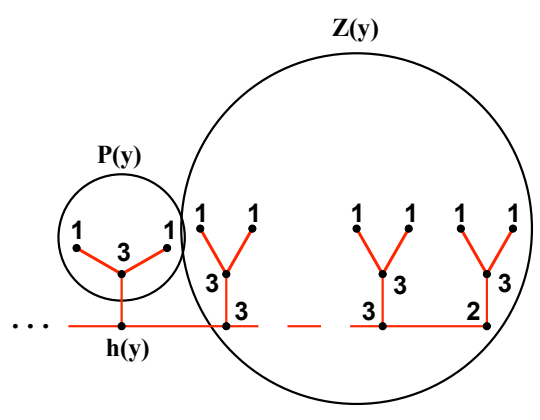

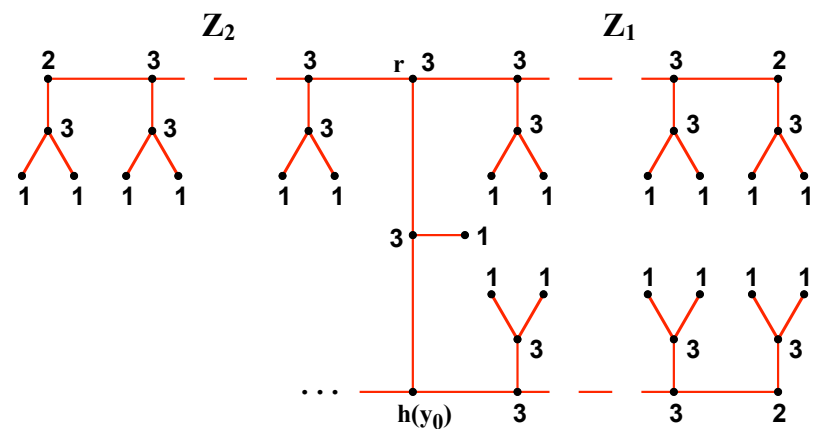

Figure 9: (a) requirement on $h(y)$, (b) $r$ connected to the multiclaws $Z_{1}$ and $Z_{2}$.

Case 3.8.1. $d_{2} \geq 3$.

Builder moves from $h\left(y_{0}\right)$ through $r$ along the spine of $Z_{1}$, assigning images to elements of $V(T)$ as adjacency warrants, until she has moved distance $d_{2}$ from $h\left(y_{0}\right)$ and reached $h\left(y_{2}\right)$. If $y_{2}$ is a leaf, she stops, if a fork, then she can proceed as above (using $y_{2}$ in place of $\left.y_{0}\right)$.

Case 3.8.2. $d_{2}=2$.

Builder moves from $h\left(y_{0}\right)$ to $r$, assigning images to elements of $V(T)$ as adjacency warrants, so that $r=h\left(y_{2}\right)$. If $y_{2}$ is a leaf, Builder stops. Otherwise, $y_{2}$ is a fork, with two successors $y_{3}$ and $y_{4}$, at distances $d_{3}$ and $d_{4}$ from $y_{2}$, respectively.

Builder now first moves along the spine of $Z_{1}$, assigning images to elements of $V(T)$ as adjacency warrants, until she has moved distance $d_{3}$ from $h\left(y_{2}\right)$ and pauses at $h\left(y_{3}\right)$. If $y_{3}$ is a leaf, then she stops, if a fork, then she can proceed as above (using $y_{3}$ in place of $\left.y_{0}\right)$.

Builder now moves along the spine of $Z_{2}$, assigning images to elements of $V(T)$ as adjacency warrants, until she has moved distance $d_{4}$ from $h\left(y_{2}\right)$ and pauses at $h\left(y_{4}\right)$. If $y_{4}$ is a leaf, then she stops, if a fork, then she can proceed as above (using $y_{4}$ in place of $\left.y_{0}\right)$.

We conclude that Builder can eventually assign images to all elements of $V(T)$ and hence can create a red copy of $T$, a contradiction. We conclude that Builder must be able to force either $M$ or $N$.

Let the vertices $c$ of $L$ and $m, n$ of $M$ be as shown in Figure 10(a).

Claim 3.9. Suppose that Builder can force $M$, and that Builder can force some (2-colored) graph $H^{\prime}$, with $v^{\prime}$ a vertex of $H^{\prime}$ having weight 2. Then Builder can force the graph shown in Figure 10(b).

Proof. The proof mirrors the proof of Claim 3.6, with $M$ replacing $X$ throughout and $L$ replacing the isolated edge $a b$. The vertices $m, n$ of $M$ and $c$ of $L$ replace the vertices $x, y$ of $X$ and $a$ of $a b$, respectively. 


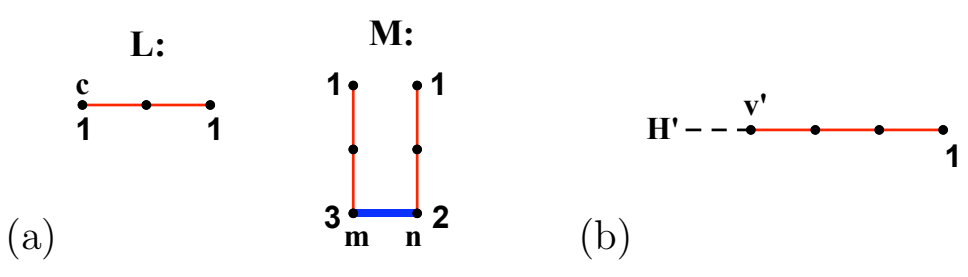

Figure 10: (a) the graphs $L$ and $M$ with vertices $c, m$, and $n$ labeled, (b) the graph desired in Claim 3.9.

Claim 3.10. Suppose that Builder can force $M$. Then, the 3-3 and 2-3 Branching Lemmas hold.

Proof. The proof of the 3-3 Branching Lemma mirrors the proof of the 2-2 Branching Lemma (following the proof of Claim 3.6), with the following modifications: (i) $M$ replaces $X$ throughout and $L$ replaces the isolated edge $a b$, with the vertices $m, n$ of $M$ and $c$ of $L$ replacing the vertices $x, y$ of $X$ and $a$ of $a b$, respectively; and (ii) invocation of Claim 3.6 is replaced by invocation of Claim 3.9.

The proof of the 2-3 Branching Lemma mirrors the proof of the 2-2 Branching Lemma without modifications up until $(*)$. At the point $(*)$, the proof continues as follows:

$(\dagger)$ If $g\left(w_{\beta}\right)$ is of the form $v$, then applying Claim 3.9 with $g\left(w_{\beta}\right)$ as $v^{\prime}$ proves the $2-3$ Branching Lemma.

If $g\left(w_{\beta}\right)$ is not of the form $v$, then $g\left(w_{\alpha}\right)$ must be of the form $v$. In this case, Builder creates a new apex, such that if $g\left(w_{\beta}\right)$ is of the form $x$ or $a$, then the new apex is of the form $m$ or $c$, respectively. Builder then connects the new apex to $g\left(w_{\alpha}\right)$ and reassigns the label $g\left(w_{\beta}\right)$ to the new apex. If the edge between $g\left(w_{\alpha}\right)$ and the new $g\left(w_{\beta}\right)$ is red, then the 2-3 Branching Lemma is proven.

Otherwise, any red-edge stoppage is moved to further along the sequence $S$. Construction of images under $g$ of elements of $S$ then proceeds as in the proof of the 2-2 Branching Lemma, except with the following modification: $M$ replaces $X$ throughout and $L$ replaces the isolated edge $a b$, with the vertices $m, n$ of $M$ and $c$ of $L$ replacing the vertices $x, y$ of $X$ and $a$ of $a b$, respectively.

If a blue $T$ is to be prevented, a red-edge stoppage next occurs between some $g\left(w_{\gamma}\right)$ and $g\left(w_{\delta}\right)$, with $\gamma<\delta$ and $\delta>\beta$. If $g\left(w_{\delta}\right)$ is of the form $v$, then applying Claim 3.6 with $g\left(w_{\delta}\right)$ as $v^{\prime}$ proves the 2-3 Branching Lemma.

If $g\left(w_{\delta}\right)$ is not of the form $v$, then $g\left(w_{\gamma}\right)$ must be of the form $v$. In this case, Builder creates a new apex, such that if $g\left(w_{\delta}\right)$ is of the form $m$ or $c$, then the new apex is of the form $x$ or $a$, respectively. Builder then connects the new apex to $g\left(w_{\gamma}\right)$ and reassigns the label $g\left(w_{\delta}\right)$ to the new apex. If the edge between $g\left(w_{\gamma}\right)$ and the new $g\left(w_{\delta}\right)$ is red, then the 2-3 Branching Lemma is proven.

Otherwise, any red-edge stoppage is moved to further along the sequence $S$. Construction of images under $g$ of elements of $S$ then proceeds as in the proof of the 2-2 Branching Lemma, with no modifications. If a blue $T$ is to be prevented, a red-edge stoppage occurs 
between some $g\left(w_{\epsilon}\right)$ and $g\left(w_{\zeta}\right)$, with $\epsilon<\zeta$ and $\zeta>\delta$. Proof proceeds as from $(\dagger)$, with $\epsilon, \zeta$ replacing $\alpha, \beta$, respectively.

Since the sequence $S$ is finite and stoppages cannot be pushed infinitely far along it, the 2-3 Branching Lemma must hold if a blue $T$ is to be prevented.

We may therefore assume that Builder can force $N$.

Suppose Builder can force a (2-colored) graph $J$ having weight-1 vertex $t$. We say that the Fork Property holds in red if Builder can force at least one of the three graphs depicted in Figure 11(a) and that the Elongation Property holds in red if Builder can force at least one of the two graphs depicted in Figure 11(b). Let graphs $Z_{1}, Z_{2}, Z_{1,1}$, $Z_{1,2}$, and $Z_{2,2}$ be as depicted in Figure 11(c). Then, say that the Fork-Fork Property holds in red if Builder can force either $Z_{1}$ or else both $Z_{1,1}$ and $Z_{1,2}$ and that the Fork-Extend Property holds in red if Builder can force either $Z_{2}$ or else both $Z_{1,2}$ and $Z_{2,2}$. Say that the Iterated Fork Property holds in red if both the Fork-Fork Property and Fork-Extend Property hold in red.

We likewise say that one of the above Properties holds in blue if Builder can force the appropriate graphs with those edges being blue which are shown as red in Figure 11.

(a)

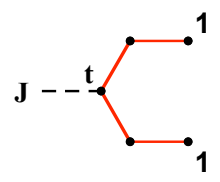

OR

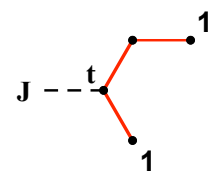

OR

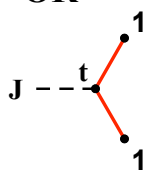

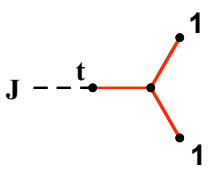

OR
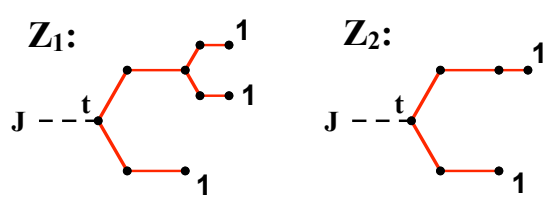

(c)

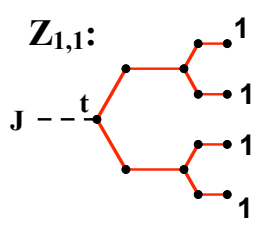

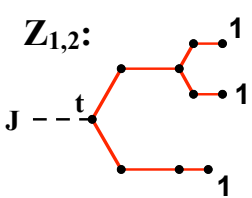

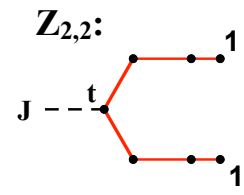

Figure 11: (a) the Fork Property, (b) the Elongation Property, (c) the graphs involved in the Iterated Fork Property.

Claim 3.11. Suppose that, for all $J$ and weight-1 $t$ that Builder can force, the Fork Property holds in blue. Then, Builder can win.

Proof. Builder maintains two trees $T_{1}$ and $T_{2}$, with $T_{1}$ red and $T_{2}$ blue, each a copy of a fitting subtree of $T$. For $i=1,2$, a certain leaf of $T_{i}$ is called the root, corresponding with a leaf of $T$; the root remains fixed even as $T_{i}$ grows. Every leaf of $T_{i}$ except the root is of weight 1 . We let $p_{i}: V\left(T_{i}\right) \rightarrow V(T)$ be the function taking vertices of $T_{i}$ to corresponding vertices of $T$. The color of $T_{i}$ is denoted $c\left(T_{i}\right)$. 
Initially, both trees are a single vertex (the root). Builder's strategy is to apply the Fork and Elongation Properties to progressively enlarge $T_{1}$ and $T_{2}$ (applying properties to $T_{i}$ in the color $\left.c\left(T_{i}\right)\right)$. Given a leaf $x$ of $T_{i}$, Builder applies the following:

- Nothing, if $p_{i}(x)$ is already a leaf of $T$.

- The Fork Property, if $p_{i}(x)$ is a fork of $T$.

- The Elongation Property, if $p_{i}(x)$ is adjacent to a fork $y$ of $T$ such that $y \notin p_{i}\left(V\left(T_{i}\right)\right)$.

- Either the Fork Property or the Elongation Property (it doesn't matter which) if $p_{i}(x)$ is not a leaf and is at distance at least 2 from any fork $y$ of $T$ such that $y \notin p_{i}\left(V\left(T_{i}\right)\right)$. In this case the whole strength of the Fork and Elongation Properties is not used, and the edges added to $T_{i}$ are either (i) a single edge in $c\left(T_{i}\right)$ starting at $x$ and with terminal vertex of weight 1 , or (ii) a path of two edges in $c\left(T_{i}\right)$ starting at $x$ and with terminal vertex of weight 1 .

The process will terminate after a finite amount of time, with $p_{i}\left(V\left(T_{i}\right)\right)$ being all of $V(T)$. It only remains to be shown that Builder can apply the Fork and Elongation Properties in the manner desired. By assumption, the Fork Property holds in blue, and by the 2-2 Branching Lemma, it holds in red. It suffices to show that at any point either Builder can apply the Elongation Property to $T_{1}$ in red or else she can apply it to $T_{2}$ in blue.

Consider $T_{1}$ and $T_{2}$ at such a point that the Elongation Property is required by $T_{1}$ at the vertex $x_{1}$ and by $T_{2}$ at the vertex $x_{2}$. Builder first constructs two edges emanating from each $x_{i}$, with the other endpoints of these edges at new vertices. If either edge at $x_{i}$ is in $c\left(T_{i}\right)$, the Elongation Property is achieved in $c\left(T_{i}\right)$ on $T_{i}$ and we are done. Otherwise, both edges at $x_{1}$ are in blue and both edges at $x_{2}$ are in red. Connecting $x_{1}$ and $x_{2}$ by an edge then forces the Elongation Property either in red on $T_{1}$ or in blue on $T_{2}$.

Claim 3.12. Suppose that, for all $J$ and weight-1 that Builder can force, either the Fork or Iterated Fork Property holds in blue. Then, Builder can win.

The proof of this claim follows closely the proof of the previous claim. The graph $Z_{1}$ depicted in Figure 11(c) can be thought of as a composite of the Fork Property with another Fork Property applied at one of the leaves of the fork. The graph $Z_{2}$ is a composite of the Fork Property with the Elongation Property applied at one of the leaves of the fork. The graphs $Z_{1,1}, Z_{1,2}$, and $Z_{2,2}$ may be described similarly as composites of the Fork Property with some combination of Fork and Elongation Properties applied at the leaves of the fork.

We now finally are able to prove the 3-3 and 2-3 Branching Lemmas.

Proof of the 3-3 Branching Lemma. Per Claim 3.11, Builder begins by forcing some $J$ and $t$ for which the Fork Property in blue does not hold. Builder also forces the $H$ and $v$ in the statement of the 3-3 Branching Lemma. Let the graphs $C_{1}, C_{2}, D_{1}, D_{2}$ be as depicted in Figure 12. 


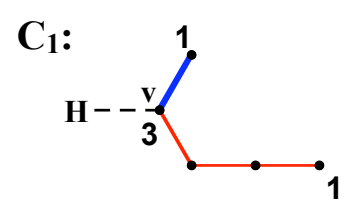

$\mathrm{C}_{2}$ :

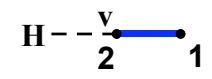

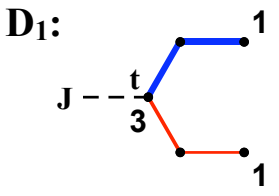

$\mathbf{D}_{2}$ :

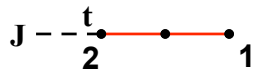

Figure 12: The graphs $C_{1}, C_{2}, D_{1}$, and $D_{2}$.

If Builder can force $C_{1}$ and $D_{1}$, then the 3-3 Branching Lemma holds, since connecting $v$ in $C_{1}$ to $t$ in $D_{1}$ yields either the Fork Property in blue at $t$ or else the 3-3 Branching Lemma at $v$. Hence, if Builder can force $C_{1}$ and $D_{2}$, then the 3-3 Branching Lemma holds, since connecting $v$ in $C_{1}$ to $t$ in $D_{2}$ yields either $D_{1}$ or else yields the 3-3 Branching Lemma at $v$. Also, if Builder can force $C_{2}$ and $D_{1}$, then the 3-3 Branching Lemma holds, since connecting $v$ in $C_{2}$ to $t$ in $D_{1}$ yields either $C_{1}$ or else yields the Fork Property in blue at $t$. We conclude that if Builder can force $C_{2}$ and $D_{2}$, then the 3-3 Branching Lemma is proven, since connecting $v$ in $C_{2}$ to $t$ in $D_{2}$ yields either $C_{1}$ or $D_{1}$. Hence, it suffices to show that Builder both can force either $C_{1}$ or $C_{2}$ and can force either $D_{1}$ or $D_{2}$.

We first prove that, if the 3-3 Branching Lemma does not hold, Builder can force either $C_{2}$ or $C_{1}$. Builder makes an edge $v w$ from $v$ in $H$ to a new vertex $w$. If $v w$ is blue, then $C_{2}$ is obtained. Otherwise, $v w$ is red, and Builder applies the Tree Extension Lemma at $w$. She then makes an edge from $v$ to a new vertex $x$. If $v x$ is blue, then $C_{1}$ is obtained. Otherwise, $v x$ is red, and Builder applies the Tree Extension Lemma at $x$, proving the 3-3 Branching Lemma.

Now, we prove that Builder can force either $D_{2}$ or $D_{1}$. Builder first makes an edge from $t$ in $J$ to the central vertex $q$ in the graph $N$ shown in Figure 8(a). If $t q$ is red, then $D_{2}$ is obtained. Otherwise, $t q$ is blue, and Builder draws a new edge from $t$ in $J$ to the vertex $q$ in a different copy of $N$. If this new edge is red, then $D_{1}$ is obtained. Otherwise, the Fork Property in blue holds at $t$.

Hence, if the 3-3 Branching Lemma does not hold, then Builder both can force $C_{1}$ or $C_{2}$ and can force $D_{1}$ or $D_{2}$, completing our proof.

Proof of the 2-3 Branching Lemma. Per Claim 3.12, Builder begins by forcing some $J$ and $t$ for which neither the Fork nor the Iterated Fork Property holds in blue at $t$. Builder also forces $H$ and $v$ as in the statement of the 2-3 Branching Lemma. Let the graphs $C_{1}, C_{2}, C_{3}, D_{1}, D_{2}, E$ be as depicted in Figure 13, with the vertex $u$ of $E$ as marked.

Builder begins by taking a copy of $H$ and constructing an edge from $v$ to a new vertex $w$. If this edge $v w$ is red, then applying the Tree Extension Lemma at $w$ yields $C_{3}$. However, if Builder can force $C_{3}$, Claim 3.6 implies that the 2-3 Branching Lemma holds. Hence, we may assume $v w$ is blue, in which case $C_{2}$ has been created.

We now prove that Builder can force $D_{2}$ or $D_{1}$. Builder first makes an edge from $t$ in $J$ to the vertex $q$ in a copy of $N$. If $t q$ is red, then $D_{2}$ is obtained. Otherwise, $t q$ is blue, 

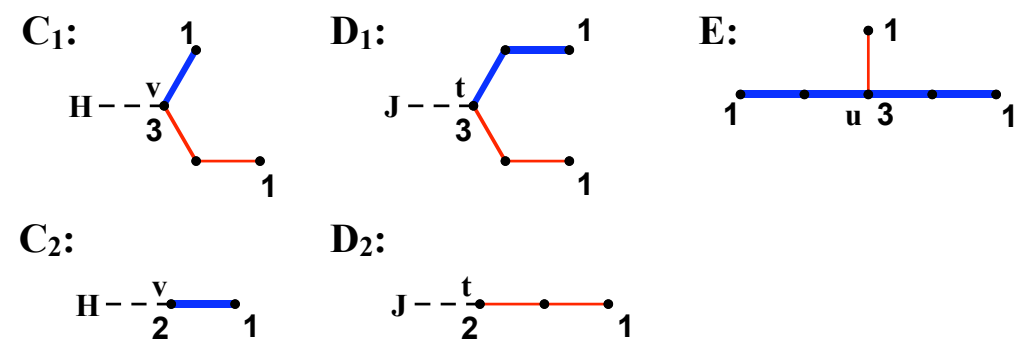

$\mathbf{D}_{2}$ :

$\mathrm{C}_{3}$ :
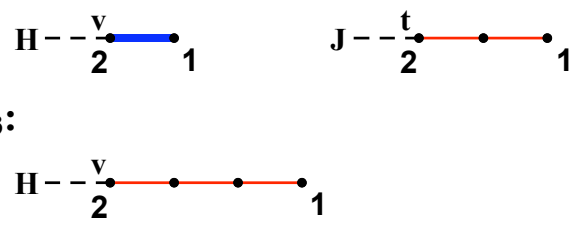

Figure 13: The graphs $C_{1}, C_{2}, C_{3}, D_{1}, D_{2}$, and $E$.

and Builder draws a new edge from $t$ in $J$ to the central vertex $q$ in a different copy of $N$. If this new edge is red, then $D_{1}$ is obtained. Otherwise, the Fork Property in blue holds at $t$.

If Builder can force $C_{1}$ and $D_{1}$, then the 2-3 Branching Lemma holds, since connecting $v$ in $C_{1}$ to $t$ in $D_{1}$ yields either the Fork Property in blue at $t$ or else the 2-3 Branching Lemma at $v$. Hence, if Builder can force $C_{1}$ and $D_{2}$, then the 2-3 Branching Lemma holds, since connecting $v$ in $C_{1}$ to $t$ in $D_{2}$ yields either $D_{1}$ or else the 2-3 Branching Lemma at $v$. Since we know Builder can force either $D_{1}$ or $D_{2}$, we conclude that if Builder can force $C_{1}$, then the 2-3 Branching Lemma holds.

Builder now forces two copies of $C_{2}$ and one isolated red edge $a b$. She proceeds to connect the vertex $a$ with the vertex $v$ in each copy of $C_{2}$. If either edge is red, then $C_{1}$ has been formed and so the 2-3 Branching Lemma holds. We may therefore assume that both edges are blue, in which case $E$ has been created.

Our strategy from here on is to prove that the Iterated Fork Property holds in blue at $t$ if the 2-3 Branching Lemma does not hold at $v$ and the Fork Property does not hold in blue at $t$. We must show that both the Fork-Fork and Fork-Extend Properties hold in blue at $t$.

Builder begins by forcing a copy of either $D_{1}$ or $D_{2}$, as well as a copy of $H$. She then connects the $v$ in the $H$ with the $t$ in the $D_{i}$. If the connecting edge is red, then $C_{3}$ has been produced and the 2-3 Branching Lemma holds. Otherwise, the edge is blue.

Builder now adds an edge from $v$ to a new vertex $s$. If $v s$ is blue, then, if $i=1$, the Fork Property holds in blue at $t$, and, if $i=2, D_{1}$ is created, allowing for a repetition of the previous steps with $i=1$. We may therefore assume that $v s$ is red. Builder applies the Tree Extension Lemma at $s$. We denote by $P$ the background graph at this point (see Figure 14(a)).

$(\ddagger)$ We now show that the Fork-Fork Property holds: namely, that Builder can force either $Z_{1}$ (as shown in Figure $11(\mathrm{c})$ ) or else both $Z_{1,1}$ and $Z_{1,2}$. Builder forces $E$ and connects the vertex $u$ in $E$ with the existing vertex $v$. If the connecting edge is red, then the 2-3 Branching Lemma holds. Otherwise, $u v$ is blue. Then, if $i=1, Z_{1}$ is forced. Suppose then that $i=2$. 

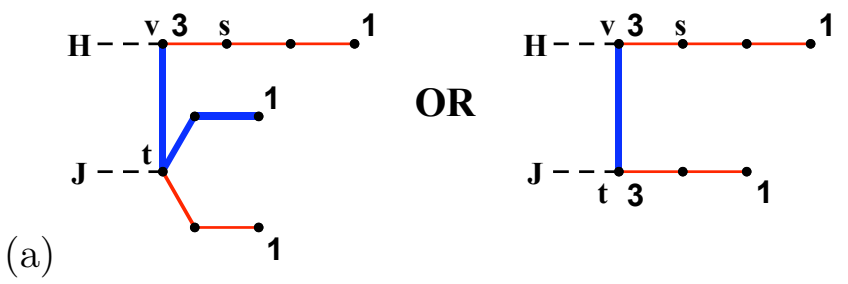

(a)

Figure 14: (a) The graph $P$ if $D_{i}$ is $D_{1}$ or $D_{2}$, respectively, (b) the graph $Q$.

Builder now forces another copy $H^{\prime}$ of $H$ and connects its copy $v^{\prime}$ of $v$ with the existing vertex $t$. If $v^{\prime} t$ is red, then $C_{3}$ is produced. Otherwise, $v^{\prime} t$ is blue. Builder now adds an edge from $v^{\prime}$ to a new vertex $s^{\prime}$. If $v^{\prime} s^{\prime}$ is blue, then $Z_{1}$ is forced. We may therefore assume $v^{\prime} s^{\prime}$ is red. Builder applies the Tree Extension Lemma at $s^{\prime}$. We denote by $Q$ the background graph at this point (see Figure 14(b)).

Builder now forces another copy $E^{\prime}$ of $E$ and connects its copy $u^{\prime}$ of $u$ with the existing vertex $v^{\prime}$. If the connecting edge is red, then the 2-3 Branching Lemma holds. Otherwise, $u^{\prime} v^{\prime}$ is blue, in which case $Z_{1,1}$ is forced.

Builder now forces another copy of $Q$ and also a copy of $N$, drawing the edge $v^{\prime} q$ between them. If $v^{\prime} q$ is red, then the 2-3 Branching Lemma holds. Otherwise, $v^{\prime} q$ is blue, in which case $Z_{1,2}$ is forced. We conclude that Builder can force either $Z_{1}$ or else both $Z_{1,1}$ and $Z_{1,2}$.

We must also show that the Fork-Extend Property holds. Builder here forces another copy of the graph $P$ and proceeds from $(\ddagger)$ with the modification that in the paragraph $(\ddagger)$ the graph $E$ is replaced by $N$ and the vertex $u$ by $q$.

We conclude that both the Fork-Fork and Fork-Extend Properties hold in blue at $t$, implying that the Iterated Fork Property must as well. This is a contradiction, and so the 2-3 Branching Lemma holds.

We have at last proven the Tree Extension, 2-2 Branching, 3-3 Branching, and 2-3 Branching Lemmas. Builder can use these lemmas to force a red copy of $T$. She does this by forcing a red copy of the maple $T^{\prime}$ obtained from $T$ by appending edges as necessary to the leaves of $T$ such that each leaf is at even distance from its nearest fork. This red copy of $T^{\prime}$ may be expressed by an injective function $k$ from $V\left(T^{\prime}\right)$ to the vertex set of the background graph of the game, such that two adjacent vertices in $V\left(T^{\prime}\right)$ are send to vertices adjacent by a red edge.

Builder defines such a function progressively over $V\left(T^{\prime}\right)$. She starts at some leaf $u$ of the maple $T^{\prime}$, assigning $k(u)$ to the vertex $c$ of a copy of the graph $L$ (see Figure 10(a)) and making the remaining vertices of $L$ be images of elements of $V\left(T^{\prime}\right)$ as adjacency warrants.

Assume that Builder has already defined $k(V(U))$ for some fitting subtree $U$ of $T^{\prime}$, 
where all leaves of $U$ have images of weight 1 . We let $p: V(U) \rightarrow V\left(T^{\prime}\right)$ be the natural inclusion function. Given a leaf $v$ of $U$ such that $p(v)$ is a fork, we let a $v$-successor be a fork or leaf that abuts $p(v)$ and is not in $p(V(U))$. Each leaf $v$ of $U$ such that $p(v)$ is a fork must have exactly two $v$-successors.

Then, given a leaf $v$ of $U$ such that $p(v)$ is not a leaf, Builder applies at $k(v)$ either the Tree Extension Lemma or one of the Branching Lemmas, as follows:

- Builder applies the Tree Extension Lemma if $p(v)$ is not a fork.

- Builder applies the 2-2 Branching Lemma if $p(v)$ is a fork and if the two $v$-successors are both at even distance from $v$.

- Builder applies the 3-3 Branching Lemma if $p(v)$ is a fork and if the two $v$-successors are both at odd distance from $v$.

- Builder applies the 2-3 Branching Lemma if $p(v)$ is a fork and if one of the two $v$-successors is at even distance from $v$ while the other is at odd distance.

After applying at $k(v)$ the Tree Extension Lemma or one of the Branching Lemmas, Builder assigns images under $k$ of elements of $V\left(T^{\prime}\right)$ as adjacency warrants.

This process ends exactly when $U=T^{\prime}$. Builder thus can create a red copy of $T^{\prime}$ and hence of $T$.

Our proof of Theorem 3.1 is finally complete. We thus have classified all trees $T$ satisfying $\stackrel{\circ}{R}_{\Delta}(T) \leq 4$. Butterfield et al. [1] described all trees $T$ satisfying $\stackrel{\circ}{R}_{\Delta}(T) \leq 3$; these trees are the paths $P_{n}$ and the claw $K_{1,3}$. Hence, we obtain immediately the following as a corollary to Theorem 3.1:

Corollary 3.13. The set of trees $T$ for which $\stackrel{\circ}{R}_{\Delta}(T)=4$ is the set of maples with the addition of the graph $K_{1,4}$ and with the removal of the claw $K_{1,3}$ and of all paths $P_{n}$.

\section{Further Questions}

It is our hope that our classification of trees $T$ satisfying $\stackrel{\circ}{R}_{\Delta}(T)=4$ will be useful in the development of a general classification of graphs $G$ which satisfy $\stackrel{\circ}{R}_{\Delta}(G)=4$. We will shortly be presenting a proof that all cycles satisfy this equation, as well as a description of many other graphs which satisfy it. However, the problem of full classification appears to us to be quite challenging.

Many other questions are open relating to on-line degree Ramsey numbers, including the following, suggested in [1]: Can graphs $G$ with maximum degree fixed at $d$ have arbitrarily large on-line degree Ramsey numbers? Additionally, the generalizations of our results and those in [1] to a game with additional colors could prove interesting. 


\section{Acknowledgments}

This research was performed at the University of Minnesota Duluth REU and was supported by the National Science Foundation (grant number DMS-0754106) and the National Security Agency (grant number H98230-06-1-0013).

I would to thank Joe Gallian for supervising this research. I am indebted to Prof. Gallian, Maria Monks, Nathan Pflueger, and the other students and participants in the Duluth REU for all the advice and support that they have given me.

\section{References}

[1] J. Butterfield, T. Grauman, B. Kinnersley, K. Milans, C. Stocker, and D. West, On-line Ramsey theory for bounded degree graphs, preprint.

[2] D. Conlon, On-line Ramsey Numbers, SIAM J. Discrete Math., 23 (2009), 1954-1963. 